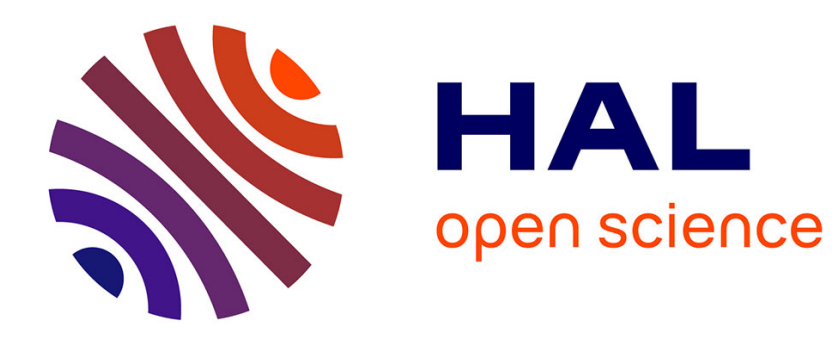

\title{
Location of Stations in a One-Way Electric Car Sharing System
}

\author{
Hatice Çalik, Bernard Fortz
}

\section{To cite this version:}

Hatice Çalik, Bernard Fortz. Location of Stations in a One-Way Electric Car Sharing System. IEEE Symposium on Computers and Communications, 2017, Heraklion, Greece. hal-01665609

\section{HAL Id: hal-01665609 \\ https://hal.inria.fr/hal-01665609}

Submitted on 21 Dec 2017

HAL is a multi-disciplinary open access archive for the deposit and dissemination of scientific research documents, whether they are published or not. The documents may come from teaching and research institutions in France or abroad, or from public or private research centers.
L'archive ouverte pluridisciplinaire HAL, est destinée au dépôt et à la diffusion de documents scientifiques de niveau recherche, publiés ou non, émanant des établissements d'enseignement et de recherche français ou étrangers, des laboratoires publics ou privés. 


\section{Location of Stations in a One-Way Electric Car Sharing System}

\author{
Hatice Çalık \\ Department of Computer Science \\ Université Libre de Bruxelles \\ 1050 Brussels, Belgium \\ E-mail: hatice.calik@ulb.ac.be
}

\author{
Bernard Fortz \\ Department of Computer Science \\ Université Libre de Bruxelles \\ 1050 Brussels, Belgium \\ E-mail: bernard.fortz@ulb.ac.be
}

\begin{abstract}
We introduce a strategic decision problem in a oneway electric car sharing system. We propose a mixed integer linear programming formulation for solving this problem. We conduct an extensive computational study to test the performance of our formulation and its relaxations by using real data instances. The results turn out to be encouraging for diving into more challenging extensions of the problem under consideration.
\end{abstract}

\section{INTRODUCTION}

The car sharing systems are based on shared use of cars owned by a company or an organization. These systems usually attract attention in urban areas with traffic congestion and high parking costs. Considering the increasing pollution in the cities, usage of electric cars in car sharing systems has a potential to increase the attractiveness of these systems [1].

In this paper, we focus on a station-based one-way electric car sharing system where we have a company that owns a fleet of identical cars available to its users who wish to travel within a region. The system is station-based as the customers have to visit some stations to pick up and drop off the cars. Most station-based systems allow only round trips where customers have to bring the cars back to the stations they have been taken from. However, this might be inconvenient for many users such as the ones who wish to travel to an airport and leave the city. One-way systems are more flexible in that sense as they allow customers to leave the cars at stations different than the ones that they are taken from.

Due to the battery restriction of the cars, location of recharging stations and management of recharging times become crucial in planning of car sharing systems. Therefore, in this study, we aim to decide on the optimal locations of recharging stations in the most profitable way for the company based on an a priori known or forecasted demand. The demand is defined as a set of customer requests and each request is associated with an origin node, a destination node, and a starting time.

A customer request can be served only under certain conditions such as accessibility to located stations, availability of a car at the departure station, and availability of an empty spot at the arrival station as the stations have only a limited number of parking spaces. We measure accessibility by walking distance, that is, a customer request is accessible if both its origin and destination nodes are within the imposed walking distance limit from some operational stations.

For simplicity reasons, we assume that a car should be fully charged to be available for the next customer. Therefore, every car left to the arrival station of a request served should be plugged into a charger and charged to full battery level. The number of charging units in a station is equal to the number of parking spots and represented as the capacity of that station.

The problem we study requires to decide on the number and location of stations and the number of cars available at each station in a way to maximize the profit which takes into account the revenue obtained from customers that can be served, the fixed location cost of stations, and the cost of purchasing or leasing cars. We formulate this problem as a mixed integer linear programming formulation (MILP) and with this MILP, we also decide on the customers that can be served, the stations they need to visit (routes), and the time of each visit simultaneously.

In the following section, we briefly review the related works in the literature. In Section III, after defining our problem explicitly, we provide the details of our MILP formulation and a pre-processing procedure that we need for our model. Finally, in Section IV, we present the results of the computational experiments that we conducted on our formulation and two relaxations of it by using real data instances.

\section{RELATED WORKS}

The majority of the existing studies on the car sharing systems focus on tactical and operational level decision problems. Among these studies, the most commonly studied problem is the relocation of cars to avoid imbalance of cars in stations. On the other hand, the literature is quite sparse in terms of the studies that focus on location of stations in car sharing systems.

The first mixed integer programming formulations for optimal location of stations in one-way car sharing system were proposed by [2]. The objective of the problem considered in this study was to maximize the profit of the company by taking into account all cost and revenue factors. The authors analyzed the model under three service strategies: (i) The operator can freely choose among the customer requests to serve (ii) all requests must be served (iii) a request can be 
rejected only if there is no car available at the starting station. The evaluation of the three models on a real data from the municipality of Lisbon, Portugal revealed that satisfying all customer requests might decrease the profit of the company significantly. These models were based on the assumption that the customers can use only the closest stations to their origin and destinations and this assumption was considered to be very restrictive by [3]. Therefore, [3] extended these formulations to a more flexible setting where more vehicle pick-up and dropoff station options were included. The case study on the same data showed that introduction of this kind of user flexibility and vehicle stock information increased the profit of company.

Other than the aforementioned optimization models, [4] provided a discrete event simulation based model that evaluates the effects of strategy changes in car sharing systems such as creating new stations, increasing station capacities, merging or demerging stations. The authors evaluated their model through several strategies on a data from a car sharing organization in Montreal, Canada.

The only study that considered location of recharging stations in an electric car sharing system was due to [5]. The authors developed a multi-objective mixed integer linear programming model that combines strategic (location of stations) and tactical (relocation of vehicles) decisions in a one-way electric car sharing system. As the model was intractable for solving realistic size problems due to the large number of relocation variables, an aggregated demand structure was used. The first objective function of the model proposed aimed to maximize the net profit of the operator whereas the second objective maximized the users' net benefit. In [5], the charging periods were assumed to be given explicitly as input parameters. The authors evaluated the performance of their approach on a real data from Nice, France.

Another group of relevant studies were on the location of charging stations for private electric vehicles. For the exact solution methodologies in this area, we refer the readers to the papers by [6], [7], [8], [9], [10], and [11]. The heuristic approaches for location of public charging units were due to [12], [13], and [14].

\section{PROPOSED FORMULATION}

Given a city network $G=(V, A)$ with arc set $A$, node set $V$, and set of potential stations $J \subset V$, we are required to select a subset $\bar{J}$ of $J$, locate an initial number of cars at the selected stations, and choose (serve) a subset $\bar{K}$ of given set of customer requests $K$. When choosing the requests, we need to make sure that each of them is served by a car available at the time of departure at a station (departure station) accessible from the origin of the request and the trip of this car ends up at another selected station (arrival station) which is accessible from the destination of the request and has a parking slot available at the time of arrival. We also make sure that the battery consumption on the way between the two stations does not exceed the battery limit $\beta$ and the total time spent between the origin and the destination, including walking time, is not greater than a given threshold $\gamma_{k}$ for request $k \in K$.
We calculate the travelling time and energy consumption for each request based on the shortest path between departure and arrival stations assigned to it. However, one can also assume that these values are given for each request as input parameters and our model still holds. Once a car completes its service and is parked to a station, its recharging starts immediately and it becomes available for another customer at the beginning of the first period after it is fully charged. Further, we need to ensure that the total number of cars waiting at a station (available or being charged) should not be greater than the capacity of the station at any point of time. The objective of the problem is to maximize the total profit that is equivalent to the total revenue obtained from the requests served minus the total cost of stations selected and cars owned.

We formulate our problem as a mixed integer linear programming formulation. Before introducing our formulation, below we provide the parameter definitions used in our formulation all together:

- $V=\{1, \ldots, n\}$ is the set of nodes.

- $J=\{1, \ldots, m\}$ is the set of potential stations where $J \subset V$.

- $f_{j}$ is the fixed cost of locating a station on vertex $j \in J$.

- $C_{j}$ is the capacity of station $j \in J$.

- $g$ is the cost of having a car.

- $T=\{0, \ldots, \tau\}$ is the set of time slots (identical length).

- $K$ is the set of requests with origin $O_{k} \in V$, destination $D_{k} \in V$, starting time $T_{k} \in T$, and revenue $p_{k}$ for $k \in K$.

- $\delta_{i j}$ is the battery usage on the way from station $i \in J$ to station $j \in J$.

- $\beta$ is the restriction on the battery usage for each car.

- $d_{i j}$ is the travel time from station $i \in J$ to station $j \in J$.

- $d_{i j}^{w}$ is the walking distance from node $i \in V$ to node $j \in S$.

- $\beta^{w}$ is the maximum walking distance between the origin (destination) points of customers and the departure (arrival) stations they are assigned to.

- $\gamma_{k}$ is the maximum length that a route assigned to customer $k$ can have.

We refer to our MILP model as a path based formulation since a set of decision variables in the model are associated with the set of feasible paths for each request. We apply a preprocessing procedure to construct this set $H_{k}$ of all feasible paths (trips) for each request $k \in K$. Let $H=\bigcup_{k \in K} H_{k}$, a trip $h \in H$ is feasible if it satisfies the accessibility and battery restrictions. For each $h \in H$, we know the stations visited on the trip $\left(P_{h}\right)$, the time of visit to each station, the amount of battery consumption, the amount of time required to recharge the car at the arrival station, thus, the time zone that the car will be ready for another customer.

Our formulation can be easily extended to solve more challenging variants of our problem by slightly modifying this pre-processing procedure. One of these extensions is the case that customers are allowed to visit intermediate stations in case the length of the trip is too long to traverse without recharging the battery or changing the car. In Algorithm 1, we provide a 
pseudo-code of this generalized case but we do not consider the exchange of cars in this study as it is unlikely to have these type of requests inside cities, which is our current focus. Another extension is to consider time dependent travelling times and energy consumptions in the problem.

Based on the information obtained from the pre-processing, we further define the following parameters:

- $b_{h j}^{t}=1$ if path $h \in H$ exits station $j \in J$ at time $t \in T$, 0 otherwise.

- $\mu_{h j}^{t}=1$ if the car used in trip $h$ to enter station $j \in J$ is being recharged at time $t \in T, 0$ otherwise.

- $\lambda_{h j}^{t}=1$ if the charging of a car used on path $h \in S_{s}$ is completed at station $j \in J$ at time $t \in T, 0$ otherwise.

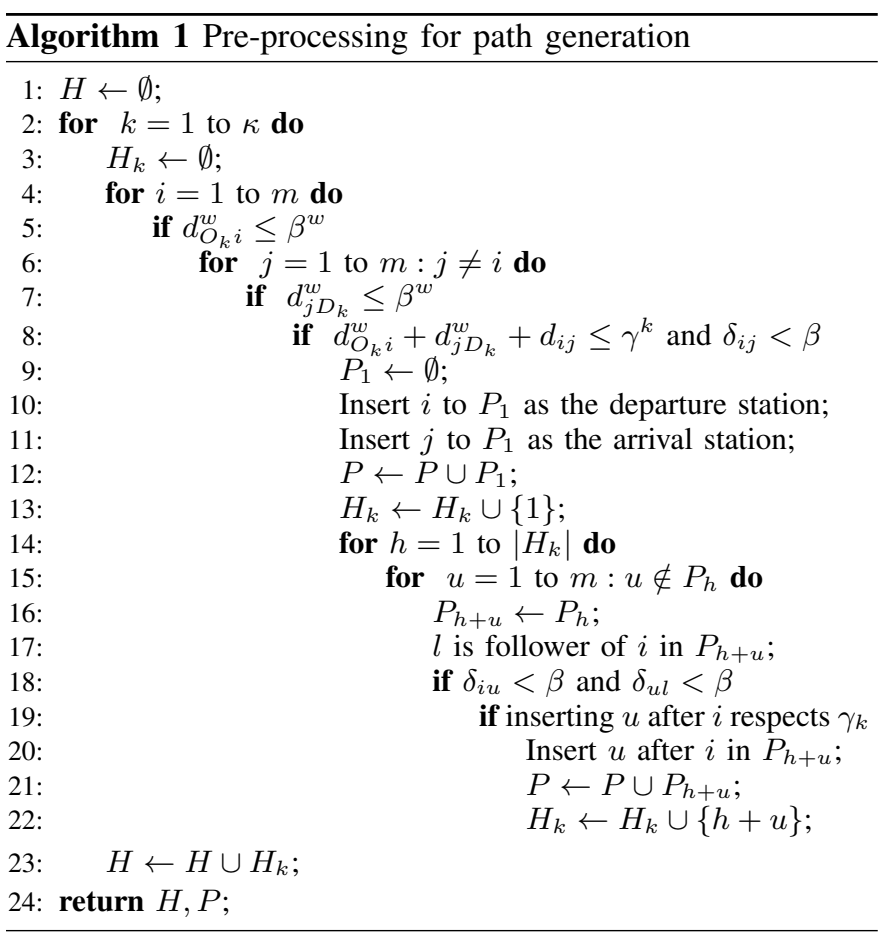

Now, we define the following decision variables to use in our formulation:

- $u_{h}=1$ if trip $h \in H$ is chosen, 0 otherwise.

- $L_{j}^{t}$ is the number of available cars at station $j \in J$ at the beginning of time $t \in T$.

- $L_{j}^{0}$ is the number of available cars at station $j \in J$ at time 0 .

- $y_{j}=1$ if a station is located at vertex $j \in I, 0$ otherwise.
Then, we can write our path based formulation (PF) as follows:

$$
\begin{aligned}
& \max \sum_{h \in H} p_{h} u_{h}-\sum_{j \in J} f_{j} y_{j}-g \sum_{j \in J} L_{j}^{0} \\
& \text { s.t. } \sum_{h \in H_{k}} u_{h} \leq 1, \quad \forall k \in K \\
& u_{h} \leq y_{j} \text {, } \\
& \forall h \in H, j \in P_{h} \\
& \sum_{h \in H} b_{h j}^{t} u_{h} \leq L_{j}^{t} \\
& L_{j}^{t}+\sum_{h \in H}\left(\mu_{h j}^{t}-b_{h j}^{t}\right) u_{h} \leq C_{j} y_{j}, \quad \forall j \in J, t \in T \\
& L_{j}^{t}=L_{j}^{(t-1)}+\sum_{h \in H}\left(\lambda_{h j}^{t}-b_{h j}^{(t-1)}\right) u_{h}, \quad \forall j \in J, t \geq 1 \\
& 0 \leq L_{j}^{t} \leq C_{j} y_{j}, \quad \forall j \in J, t \in T \\
& L_{j}^{0} \in \mathbb{Z}^{+}, \quad \forall j \in J \\
& u_{h} \in\{0,1\}, \quad \forall h \in H \\
& y_{j} \in\{0,1\} \text {, }
\end{aligned}
$$

The objective function (1) maximizes the profit. The first term in this function gives the expected revenue obtained by serving the customers, the second term is the total fixed cost of opened stations, and the third term is total cost of car ownership. By Constraints (2), a customer is served with at most one trip and by Constraints (3) every station on a selected trip is forced to be opened. Constraints (4) restrict the number of cars leaving a station with the number of available cars at that station for each time slot. Constraints (5) ensure that the capacity of each station is respected, so that parking a car is not allowed if there is no free space at the station. Constraints (6) balance the number of cars at each station at each time slot and Constraints (7) restrict this number with the capacity of that station. Finally, Constraints (8)-(10) represent the integrality and binary restrictions.

\section{COMPUTATIONAL RESUlts}

We tested our model PF, its linear programming relaxation $\mathrm{LP}$, and a partial relaxation RPF (PF where binary restrictions on $u_{h}$ is replaced with $0 \leq u_{h} \leq 1, \forall h \in H$ ) on instances obtained from Manhattan taxi trips data. This data file is based on a city network in Manhattan (New York, USA) with 10556 nodes, 85 potential station locations with non-identical capacities, 25592 edges, and 27549 requests. It contains the distance and time dependent maximum travelling speed for each edge; cost values for stations, installation of slow or fast charging units, and purchase of three types of electric cars; charging speed of slow and fast charging units; and profit of individual requests. Therefore, we are able to obtain all the parametric values we need in our calculations, including travelling time and energy consumption on each edge and recharging time after each trip, from this data file. 
We chose a single car type, fast charging as the charging type, and 5 mins as the length of each period. We computed the shortest paths for walking and traveling times by using Dijkstra's algorithm.

We conducted our experiments by using IBM ILOG CPLEX 12.7 on an Intel Core i7-4510U processor with CPU at 2.00 GHz and 8 GB RAM. For the Dijkstra's algorithm, we used the implementation of the JGrapghT 1.0.1 package.

In order to balance the cost and profit values and avoid trivial solutions with 'do nothing' decision, we divided the station costs and car cost with a cost factor. This value can also be considered as a factor to represent the expected frequency of similar requests in the long run. In our experiments, we used three different cost factor values, namely, $10^{4}, 10^{5}, 10^{6}$.

In Table I, we summarize the pre-processing results for each problem instance solved. In this table, column $|K|$ gives the number of requests taken from the data and column $\left|K_{a}\right|$ gives the number of requests accessible from stations within the walking time limit (in minutes) indicated under the column of $\beta^{w}$. The values under $|H|$ show the number of paths generated during the pre-processing and 'PP Time' shows the time consumed for the pre-processing (in seconds).

In Tables II, III, and IV, we detail the structural results deducted from the solutions for cost factor values $10^{4}, 10^{5}$, and $10^{6}$, respectively. Let 'LP Opt', 'RPF Opt', and 'PF Opt' provide the optimal solution values obtained from LP, RPF, and PF, respectively. Then, the 'LP gap' and 'RPF gap' values in Tables II, III, and IV are calculated with the formulations $(100 *($ LP Opt - PF Opt)/PF Opt) and $(100 *(\mathrm{RPF}$ Opt $-\mathrm{PF}$ Opt)/PF Opt), respectively. In these tables, the columns under $|\bar{J}|$ provide the number of stations opened and $|\bar{H}|$ represents the number of trips, thus, requests served in the corresponding optimal solution of PF.

We observe from Tables II, III, and IV that the optimal values of RPF and PF agree in each instance. Here we note that the RPF solutions, that is, a subset of $u_{h}, h \in H$ variables are fractional in some of the instances. As expected, the number of trips served and the profit obtained increase as $\beta^{w}$ is increased. This increases also the size of the problem and the time consumed during the pre-processing and total time for solving the problem.

In most of the instances, we observe a very small LP gap. Among the 63 instances, only four of them have an LP gap greater than $10 \%$ and the average LP gap is around $2.5 \%$.

When we look at the impact of the change in the cost factor value, we see that the model has a tendency to open more stations and serve more customers when the cost factor is higher (i.e. when the long term profit of each request is higher). The increase is more significant and the impact is more visible when the change is from $10^{4}$ to $10^{5}$ compared to the case where it is from $10^{5}$ to $10^{6}$. In Table II, PF decides not to open any stations for three of the instances as it is not profitable. Among these three instances, the LP solution is non-trivial only for the one with $|K|=1000, \beta^{w}=6$ and LP $\mathrm{Opt}=135.00$ for this problem. We see in this table that the model is conservative in the sense that it does not open a large
TABLE I: Pre-processing results

\begin{tabular}{|r|r|r|r|r|}
\hline$|K|$ & $\beta^{w}$ & $\left|K_{a}\right|$ & $|H|$ & PP Time \\
\hline 1000 & 5 & 53 & 72 & 2.72 \\
1000 & 6 & 85 & 170 & 3.09 \\
1000 & 7 & 121 & 274 & 5.91 \\
1000 & 8 & 174 & 432 & 5.88 \\
1000 & 10 & 314 & 1033 & 12.04 \\
1000 & 15 & 718 & 5212 & 44.02 \\
\hline 2000 & 5 & 91 & 130 & 3.24 \\
2000 & 6 & 162 & 313 & 5.38 \\
2000 & 7 & 245 & 555 & 7.19 \\
2000 & 8 & 367 & 952 & 10.56 \\
2000 & 10 & 648 & 2360 & 19.52 \\
2000 & 15 & 1447 & 11756 & 101.56 \\
\hline 3000 & 5 & 134 & 199 & 4.61 \\
3000 & 10 & 983 & 3686 & 29.48 \\
3000 & 15 & 2160 & 17362 & 142.80 \\
\hline 5000 & 5 & 220 & 345 & 7.3 \\
5000 & 10 & 1667 & 6254 & 65.84 \\
5000 & 15 & 3605 & 29222 & 249.30 \\
\hline 10000 & 5 & 471 & 787 & 11.16 \\
10000 & 10 & 3555 & 13531 & 100.58 \\
10000 & 15 & 7296 & 63721 & 449.10 \\
\hline
\end{tabular}

number of stations if $\left|K_{a}\right|$ is small, more specifically, the ratio of opened stations is less than $50 \%$ when $\left|K_{a}\right| \leq 314$ in this table. In Tables III and IV, we obtain optimal solutions with positive profit, which serve more than $50 \%$ of the accessible requests, for each problem. The LP gap is less than $2.4 \%$ for each instance in Table III and it is less than $0.15 \%$ in Table IV.

When we look at the solving times in Tables V, VI, and VII, we see that we are able to solve each problem in less than half an hour except one with a total solving time of 1808.38 seconds. Moreover, we observe that the time required to solve the LP is usually less than the one for PF whereas the solving time of RPF is larger than that of PF. This is, in fact, also reflected to the average solving times in Tables V and VII, but not in Table VI due to the single instance $\left(|K|=5000, \beta^{w}=\right.$ 15) where the PF time is significantly larger than the RPF time.

\section{CONClusion}

Introduction of electric cars to car sharing systems brings new challenges in strategic and operational level planning of these systems. In this paper, we introduce a strategic level problem that focuses on optimal location of charging stations in a one-way electric car sharing system. We proposed a path based mathematical formulation that can be easily adapted to solve the problem under several different settings. The computational experiments that we conducted on real data instances revealed that our model can solve quite large problems in reasonable amount of time. This is encouraging for investigating more complex generalizations and extensions of the problem. One of the extensions that would be relevant is to consider demand uncertainty. This could be achieved by introducing multiple demand scenarios generated based on the existing demand pattern. This problem is currently in our research agenda. 
TABLE II: Results for cost factor $=10^{4}$ - gaps \& solutions

\begin{tabular}{|r|r|r|r|r|r|r|}
\hline$|K|$ & $\beta^{w}$ & PF Opt & LP gap & RP gap & $|\bar{J}|$ & $|\bar{K}|$ \\
\hline 1000 & 5 & 0 & -- & -- & 0 & 0 \\
1000 & 6 & 0 & -- & -- & 0 & 0 \\
1000 & 7 & 1640 & 0.00 & 0.00 & 6 & 12 \\
1000 & 8 & 12580 & 6.35 & 0.00 & 25 & 60 \\
1000 & 10 & 63710 & 7.12 & 0.00 & 39 & 170 \\
1000 & 15 & 233080 & 3.43 & 0.00 & 50 & 432 \\
\hline 2000 & 5 & 0 & -- & -- & 0 & 0 \\
2000 & 6 & 1470 & 38.29 & 0.00 & 12 & 24 \\
2000 & 7 & 17140 & 21.89 & 0.00 & 41 & 112 \\
2000 & 8 & 61960 & 8.57 & 0.00 & 50 & 203 \\
2000 & 10 & 183770 & 4.10 & 0.00 & 56 & 407 \\
2000 & 15 & 554000 & 0.93 & 0.00 & 55 & 944 \\
\hline 3000 & 5 & 1920 & 20.83 & 0.00 & 11 & 24 \\
3000 & 10 & 318320 & 2.91 & 0.00 & 61 & 601 \\
3000 & 15 & 884180 & 0.49 & 0.00 & 58 & 1401 \\
\hline 5000 & 5 & 14560 & 10.50 & 0.00 & 33 & 89 \\
5000 & 10 & 594840 & 0.64 & 0.00 & 67 & 1045 \\
5000 & 15 & 1536670 & 0.20 & 0.00 & 63 & 2374 \\
\hline 10000 & 5 & 84040 & 1.63 & 0.00 & 58 & 285 \\
10000 & 10 & 1266930 & 0.15 & 0.00 & 70 & 2057 \\
10000 & 15 & 2941320 & 0.04 & 0.00 & 68 & 5430 \\
\hline Avg.: & & & 7.11 & 0.00 & & \\
Max: & & & 38.29 & 0.00 & & \\
\hline
\end{tabular}

TABLE III: Results with cost factor $=10^{5}$ - gaps \& solutions

\begin{tabular}{|r|r|r|r|r|r|r|}
\hline$|K|$ & $\beta^{w}$ & PF Opt & LP gap & RP gap & $|\bar{J}|$ & $|\bar{K}|$ \\
\hline 1000 & 5 & 21960 & 0.00 & 0.00 & 49 & 48 \\
1000 & 6 & 36777 & 2.39 & 0.00 & 55 & 73 \\
1000 & 7 & 53331 & 1.25 & 0.00 & 58 & 95 \\
1000 & 8 & 82107 & 0.87 & 0.00 & 62 & 134 \\
1000 & 10 & 149372 & 0.51 & 0.00 & 60 & 225 \\
1000 & 15 & 336067 & 0.11 & 0.00 & 65 & 472 \\
\hline 2000 & 5 & 37254 & 0.67 & 0.00 & 66 & 78 \\
2000 & 6 & 68433 & 1.12 & 0.00 & 69 & 128 \\
2000 & 7 & 111370 & 0.16 & 0.00 & 72 & 188 \\
2000 & 8 & 169051 & 0.39 & 0.00 & 73 & 268 \\
2000 & 10 & 295923 & 0.31 & 0.00 & 70 & 449 \\
2000 & 15 & 670332 & 0.04 & 0.00 & 67 & 988 \\
\hline 3000 & 5 & 60841 & 0.40 & 0.00 & 69 & 112 \\
3000 & 10 & 442950 & 0.09 & 0.00 & 73 & 639 \\
3000 & 15 & 1008386 & 0.02 & 0.00 & 71 & 1429 \\
\hline 5000 & 5 & 103888 & 0.22 & 0.00 & 75 & 180 \\
5000 & 10 & 730822 & 0.02 & 0.00 & 76 & 1067 \\
5000 & 15 & 1667991 & 0.01 & 0.00 & 73 & 2424 \\
\hline 10000 & 5 & 205413 & 0.11 & 0.00 & 79 & 340 \\
10000 & 10 & 1409869 & 0.02 & 0.00 & 78 & 2073 \\
10000 & 15 & 3079927 & 0.00 & 0.00 & 77 & 4493 \\
\hline Avg.: & & & 0.41 & 0.00 & & \\
Max: & & & 2.39 & 0.00 & & \\
\hline
\end{tabular}

\section{ACKNOWLEDGMENT}

This research is conducted under e4-share (Models for Ecological, Economical, Efficient, Electric Car-Sharing) project funded by FFG, INNOVIRIS and MIUR via JPI Urban Europe.

\section{REFERENCES}

[1] J. Firnkorn and M. Müller, "Free-floating electric carsharing-fleets in smart cities: The dawning of a post-private car era in urban environments?" Environmental Science \& Policy, vol. 45, pp. 30-40, 2015.

[2] G. H. Correia and A. P. Antunes, "Optimization approach to depot location and trip selection in one-way carsharing systems," Transportation Research Part E: Logistics and Transportation Review, vol. 48, no. 1, p. 233247, Jan. 2012.
TABLE IV: Results with cost factor $=10^{6}$ - gaps \& solutions

\begin{tabular}{|r|r|r|r|r|r|r|}
\hline$|K|$ & $\beta^{w}$ & PF Opt & LP gap & RP gap & $|\bar{J}|$ & $|\bar{K}|$ \\
\hline 1000 & 5 & 31071 & 0.0000 & 0.00 & 52 & 49 \\
1000 & 6 & 47099 & 0.1440 & 0.00 & 57 & 74 \\
1000 & 7 & 64367 & 0.0673 & 0.00 & 59 & 95 \\
1000 & 8 & 93735 & 0.0730 & 0.00 & 63 & 134 \\
1000 & 10 & 160603 & 0.0662 & 0.00 & 62 & 225 \\
1000 & 15 & 348158 & 0.0155 & 0.00 & 67 & 472 \\
\hline 2000 & 5 & 48754 & 0.0390 & 0.00 & 66 & 78 \\
2000 & 6 & 81132 & 0.0970 & 0.00 & 72 & 129 \\
2000 & 7 & 124501 & 0.0108 & 0.00 & 75 & 188 \\
2000 & 8 & 182680 & 0.0316 & 0.00 & 73 & 268 \\
2000 & 10 & 309110 & 0.0302 & 0.00 & 71 & 449 \\
2000 & 15 & 683370 & 0.0046 & 0.00 & 69 & 1022 \\
\hline 3000 & 5 & 73126 & 0.0431 & 0.00 & 73 & 114 \\
3000 & 10 & 457034 & 0.0097 & 0.00 & 73 & 639 \\
3000 & 15 & 1022315 & 0.0012 & 0.00 & 73 & 1429 \\
\hline 5000 & 5 & 117411 & 0.0304 & 0.00 & 75 & 180 \\
5000 & 10 & 745746 & 0.0017 & 0.00 & 77 & 1068 \\
5000 & 15 & 1682378 & 0.0007 & 0.00 & 73 & 2400 \\
\hline 10000 & 5 & 220437 & 0.0106 & 0.00 & 80 & 340 \\
10000 & 10 & 1425175 & 0.0019 & 0.00 & 78 & 2091 \\
10000 & 15 & 3095668 & 0.0002 & 0.00 & 79 & 4494 \\
\hline Avg.: & & & 0.03 & 0.00 & & \\
Max: & & & 0.14 & 0.00 & & \\
\hline
\end{tabular}

TABLE V: Solving times (sec) for cost factor $=10^{4}$

\begin{tabular}{|r|r|r|r|r|r|}
\hline$|K|$ & $\beta^{w}$ & LP & RPF & PF & PF+PP \\
\hline 1000 & 5 & 0.49 & 0.51 & 0.61 & 3.33 \\
1000 & 6 & 0.84 & 0.66 & 0.67 & 3.76 \\
1000 & 7 & 1.14 & 0.78 & 0.98 & 6.89 \\
1000 & 8 & 0.84 & 2.26 & 1.30 & 7.18 \\
1000 & 10 & 2.11 & 3.58 & 1.55 & 13.59 \\
1000 & 15 & 7.71 & 41.93 & 26.8 & 70.82 \\
\hline 2000 & 5 & 0.96 & 0.88 & 1.08 & 4.32 \\
2000 & 6 & 1.48 & 1.16 & 1.08 & 6.46 \\
2000 & 7 & 1.93 & 2.47 & 2.92 & 10.11 \\
2000 & 8 & 2.74 & 3.57 & 4.12 & 14.68 \\
2000 & 10 & 5.79 & 6.98 & 4.32 & 23.84 \\
2000 & 15 & 30.22 & 528.65 & 180.58 & 282.14 \\
\hline 3000 & 5 & 1.56 & 1.96 & 1.46 & 6.07 \\
3000 & 10 & 10.90 & 37.86 & 16.02 & 45.50 \\
3000 & 15 & 52.37 & 618.72 & 164.28 & 307.08 \\
\hline 5000 & 5 & 2.53 & 2.93 & 3.88 & 11.18 \\
5000 & 10 & 40.05 & 81.19 & 38.46 & 104.30 \\
5000 & 15 & 139.62 & 1991.69 & 304.41 & 553.71 \\
\hline 10000 & 5 & 7.27 & 7.24 & 6.83 & 17.99 \\
10000 & 10 & 80.64 & 140.52 & 104.48 & 205.06 \\
10000 & 15 & 486.66 & 3598.35 & 1359.28 & 1808.38 \\
\hline Avg.: & & 41.80 & 336.85 & 105.96 & 166.97 \\
Max: & & 486.66 & 3598.35 & 1359.28 & 1808.38 \\
\hline & & & & & \\
\hline
\end{tabular}

[3] G. H. Correia, D. R. Jorge, and D. M. Antunes, "The added value of accounting for users' flexibility and information on the potential of a station-based one-way car-sharing system: An application in Lisbon, Portugal," Journal of Intelligent Transportation Systems, vol. 18, no. 3, p. 299308, Jun. 2014.

[4] A. E. Fassi, A. Awasthi, and M. Viviani, "Evaluation of carsharing network's growth strategies through discrete event simulation," Expert Systems with Applications, vol. 39, no. 8, p. 66926705, Jun. 2012.

[5] B. Boyac1, K. G. Zografos, and N. Geroliminis, "An optimization framework for the development of efficient one-way car-sharing systems," European Journal of Operational Research, vol. 240, no. 3, p. 718733 , Feb. 2015.

[6] I. Frade, A. Ribeiro, G. Gonçalves, and A. P. Antunes, "Optimal location of charging stations for electric vehicles in a neighborhood in lisbon, Portugal," Transportation Research Record: Journal of the Transportation Research Board, vol. 2252, pp. 91-98, Dec. 2011.

[7] J. Cavadas, G. H. Correia, and J. Gouveia, "A mip model for locating slow-charging stations for electric vehicles in urban areas accounting 
TABLE VI: Solving times (sec) for cost factor $=10^{5}$

\begin{tabular}{|r|r|r|r|r|r|}
\hline$|K|$ & $\beta^{w}$ & LP & RPF & PF & PF+PP \\
\hline 1000 & 5 & 0.95 & 0.57 & 0.58 & 3.30 \\
1000 & 6 & 1.17 & 0.80 & 0.97 & 4.06 \\
1000 & 7 & 1.31 & 1.32 & 1.25 & 7.16 \\
1000 & 8 & 1.17 & 1.68 & 1.69 & 7.57 \\
1000 & 10 & 1.99 & 2.24 & 1.55 & 13.59 \\
1000 & 15 & 11.15 & 23.95 & 18.2 & 62.22 \\
\hline 2000 & 5 & 1.57 & 1.22 & 1.14 & 4.38 \\
2000 & 6 & 1.82 & 2.88 & 1.62 & 7.00 \\
2000 & 7 & 2.00 & 2.29 & 2.88 & 10.07 \\
2000 & 8 & 3.03 & 3.78 & 3.47 & 14.03 \\
2000 & 10 & 5.47 & 7.75 & 5.06 & 24.58 \\
2000 & 15 & 37.50 & 155.93 & 64.70 & 166.26 \\
\hline 3000 & 5 & 1.88 & 2.32 & 1.20 & 5.81 \\
3000 & 10 & 8.82 & 13.35 & 9.79 & 39.27 \\
3000 & 15 & 46.8 & 171.06 & 113.15 & 255.95 \\
\hline 5000 & 5 & 2.42 & 2.40 & 3.54 & 9.54 \\
5000 & 10 & 21.36 & 23.78 & 22.77 & 80.79 \\
5000 & 15 & 112.99 & 278.73 & 614.35 & 828.71 \\
\hline 10000 & 5 & 6.64 & 5.98 & 7.12 & 18.65 \\
10000 & 10 & 65.40 & 82.82 & 62.84 & 158.14 \\
10000 & 15 & 461.67 & 643.05 & 613.07 & 1075.76 \\
\hline Avg.: & & 37.96 & 68.00 & 73.85 & 133.18 \\
Max: & & 461.67 & 643.05 & 614.35 & 1075.76 \\
\hline & & & & & \\
\hline
\end{tabular}

TABLE VII: Solving times (sec) for cost factor $=10^{6}$

\begin{tabular}{|r|r|r|r|r|r|}
\hline$|K|$ & $\beta^{w}$ & LP & RPF & PF & PF+PP \\
\hline 1000 & 5 & 0.59 & 0.43 & 0.66 & 3.38 \\
1000 & 6 & 0.77 & 1.01 & 0.73 & 3.82 \\
1000 & 7 & 0.94 & 0.92 & 0.99 & 6.90 \\
1000 & 8 & 0.88 & 2.05 & 1.18 & 7.06 \\
1000 & 10 & 1.98 & 1.77 & 1.57 & 13.61 \\
1000 & 15 & 6.72 & 11.65 & 10.92 & 54.94 \\
\hline 2000 & 5 & 0.93 & 0.81 & 0.85 & 4.09 \\
2000 & 6 & 1.31 & 1.06 & 1.05 & 6.43 \\
2000 & 7 & 1.50 & 2.58 & 2.36 & 9.55 \\
2000 & 8 & 2.24 & 3.00 & 2.20 & 12.76 \\
2000 & 10 & 4.68 & 4.35 & 4.96 & 24.48 \\
2000 & 15 & 29.34 & 120.12 & 38.65 & 140.21 \\
\hline 3000 & 5 & 1.80 & 1.17 & 1.70 & 6.31 \\
3000 & 10 & 8.06 & 10.57 & 9.66 & 39.14 \\
3000 & 15 & 49.19 & 120.21 & 97.45 & 240.25 \\
\hline 5000 & 5 & 2.44 & 2.50 & 3.95 & 9.95 \\
5000 & 10 & 24.84 & 26.93 & 22.58 & 80.60 \\
5000 & 15 & 155.91 & 694.43 & 355.22 & 569.58 \\
\hline 10000 & 5 & 7.02 & 7.52 & 6.13 & 17.66 \\
10000 & 10 & 82.22 & 95.68 & 80.63 & 175.93 \\
10000 & 15 & 523.40 & 1003.95 & 965.22 & 1427.91 \\
\hline Avg.: & & 43.18 & 100.61 & 76.60 & 135.93 \\
Max: & & 523.40 & 1003.95 & 965.22 & 1427.91 \\
\hline & & & & & \\
\hline
\end{tabular}

for driver tours," Transportation Research Part E: Logistics and Transportation Review, vol. 75, pp. 188-201, Mar. 2015.

[8] Y.-W. Wang and C.-C. Lin, "Locating multiple types of recharging stations for battery-powered electric vehicle transport," Transportation Research Part E: Logistics and Transportation Review, vol. 58, pp. 7687, Nov. 2013.

[9] F. Baouche, R. Billot, R. Trigui, and N.-E. El Faouzi, "Efficient allocation of electric vehicles charging stations: Optimization model and application to a dense urban network," IEEE Intelligent Transportation Systems Magazine, vol. 6, no. 3, p. 3343, 2014.

[10] T. D. Chen, K. M. Kockelman, M. Khan et al., "The electric vehicle charging station location problem: a parking-based assignment method for seattle," in 92nd Annual Meeting of the Transportation Research Board. Washington DC, USA, 2013. [Online]. Available: http://citeseerx.ist.psu.edu/viewdoc/summary?doi=10.1.1.300.2883

[11] J. González, R. Alvaro, C. Gamallo, M. Fuentes, J. Fraile-Ardanuy, L. Knapen, and D. Janssens, "Determining electric vehicle charging point locations considering drivers' daily activities," Procedia Computer
Science, vol. 32, no. 0, pp. 647-654, 2014, the 5th International Conference on Ambient Systems, Networks and Technologies (ANT-2014), the 4th International Conference on Sustainable Energy Information Technology (SEIT-2014). [Online]. Available: http://www.sciencedirect.com/science/article/pii/S1877050914006723

[12] S. Ge, L. Feng, and H. Liu, "The planning of electric vehicle charging station based on grid partition method," in 2011 International Conference on Electrical and Control Engineering (ICECE). IEEE, Sep. 2011.

[13] A. Hess, F. Malandrino, M. B. Reinhardt, C. Casetti, K. A. Hummel, and J. M. Barceló-Ordinas, "Optimal deployment of charging stations for electric vehicular networks," in Proceedings of the First Workshop on Urban Networking, ser. UrbaNe '12. New York, NY, USA: ACM, 2012, pp. 1-6.

[14] H. Wang, Q. Huang, C. Zhang, and A. Xia, "A novel approach for the layout of electric vehicle charging station," in The 2010 International Conference on Apperceiving Computing and Intelligence Analysis Proceeding. IEEE, Dec. 2010. 